\title{
A Polarizable Electrostatic Model of the N-Methylacetamide Dimer
}

\author{
B. MANNFORS, N. G. MIRKIN, K. PALMO, S. KRIMM \\ Biophysics Research Division and Department of Physics, University of Michigan, Ann Arbor, \\ Michigan 48109
}

Received 5 December 2000; accepted 24 April 2001

\begin{abstract}
Our previously developed polarizable electrostatic model is applied to isolated N-methylacetamide (NMA) and to three hydrogen-bonded configurations of the NMA dimer. Two versions of the model are studied. In the first one (POL1), polarizability along the valence bonds is described by induced bond charge increments, and polarizability perpendicular to the bonds is described by cylindrically isotropic induced atomic dipoles. In the other version (POL2), the induced bond charge increments are replaced by induced atomic dipoles along the bonds. The parameterization is done by fitting to ab initio MP2/6-31++G(d,p) electric potentials. The polarizability parameters are determined by subjecting the NMA molecule to various external electric fields. POL1 turns out to be easier to optimize than POL2. Both models reproduce well the $a b$ initio electric potentials, molecular dipole moments, and molecular polarizability tensors of the monomer and the dimers. Nonpolarizable models are also investigated. The results show that polarization is very important for reproducing the electric potentials of the studied dimers, indicating that this is also the case in hydrogen bonding between peptide groups in proteins. (C) 2001 John Wiley \& Sons, Inc. J Comput Chem 22: 1933-1943, 2001
\end{abstract}

Keywords: N-methylacetamide; molecular mechanics; electric potential; polarization; hydrogen bond 


\section{Introduction}

C onfidence in the predictions of computational simulations on macromolecules depends on overcoming the uncertainty that "we still cannot be sure of the full adequacy of the force field." ${ }^{1}$ Our approach to achieve such "full adequacy" rests on at least two pillars: inclusion of spectroscopic accuracy (as well as structure and energy agreement) as a criterion for parameterizing a molecular mechanics (MM) force field, and incorporation of polarization (in addition to atomic charges and dipoles) in characterizing electrostatic interactions. In both cases, we use the results of high-level ab initio calculations on model molecules as the goal to be reproduced by the MM function.

Accuracy in the reproduction of vibrational normal modes is achieved by our development of what we call a spectroscopically determined force field (SDFF). In this approach, ${ }^{2}$ the basic ab initio "data" are obtained on multiple conformers of model molecules of the macromolecule, with force constants scaled to reproduce experimental frequencies and band assignments. The parameterization starts with initial nonbonded parameters (subsequently optimized $^{3}$ ) and an analytical transformation of the "data" into the MM force field for each conformer, ${ }^{2}$ followed by the optimization to a single (reduced) set of intrinsic force constants and geometry parameters. We have implemented this approach to produce SDFFs for saturated ${ }^{4,5}$ and unsaturated ${ }^{6}$ hydrocarbon chains, with reproduction of frequencies in the range of root-meansquare (rms) deviations of $5-10 \mathrm{~cm}^{-1}$.

Because the SDFF procedure depends on the assumption of an initial nonbonded potential, we have also concentrated on obtaining the most accurate representation of this part of the force field, comprising the van der Waals and electrostatic interactions. Our approach is to determine the electrostatic parameters first, because these can be obtained independently and accurately through the electric potential (unit = volt), which is closely related to the electrostatic potential energy (unit $=\mathrm{kcal} / \mathrm{mol}$ ). Our model consists of atomic charges and dipoles, and polarization is incorporated through induced atomic charges and anisotropically induced atomic dipoles. ${ }^{7}$ This model has been applied to water and formaldehyde molecules and a few hydrogen-bonded configurations of the water dimer and the water-formaldehyde complex, ${ }^{7}$ and gives a very satisfactory represen- tation of the electric potentials, molecular dipole moments, and molecular polarizabilities.

In this article, we apply our electrostatic polarizability model to the trans-N-methylacetamide (NMA) molecule and to three hydrogen-bonded configurations of the NMA dimer. As will be seen, incorporation of polarization gives a very satisfactory description of dimer properties based on monomer parameters. It has been widely recognized that nonadditive electrostatic interactions are significant, and therefore, that it is important to include polarization effects in the MM function. This has been done in various ways. The most common model is based on isotropic atomic polarizability, but other schemes have also been used, such as the fluctuating charge model by Rick et al. ${ }^{9}$ (later extended by Stern et al. ${ }^{10}$ to also include atomic polarizability), and the automated model recently proposed by Ma et al. ${ }^{11}$ that uses induced point dipoles at the midpoints of bonds. Still another model ${ }^{12}$ simulates induced atomic dipoles by modifying the values of some of the atomic charges, thereby avoiding the explicit use of atomic dipoles. Because our approach ${ }^{7}$ is somewhat different from the previous ones, we first briefly review our model. Incorporation of polarizability will ultimately lead to a more fully adequate force field, viz., a spectroscopically determined polarizable force field (SDPFF).

\section{Electrostatic Model}

The electrostatic potential energy, $V_{\mathrm{el}}$, is described in terms of atomic charges and dipoles, and is given by

$$
\begin{aligned}
V_{\mathrm{el}}= & \frac{1}{4 \pi \varepsilon_{0} \kappa}\left[\frac{q_{i} q_{j}}{R_{i j}}+\frac{q_{i} \boldsymbol{\mu}_{j} \cdot \mathbf{R}_{i j}}{R_{i j}^{3}}\right. \\
& \left.+\frac{R_{i j}^{2} \boldsymbol{\mu}_{i} \cdot \boldsymbol{\mu}_{j}-3\left(\mathbf{R}_{i j} \cdot \boldsymbol{\mu}_{i}\right)\left(\mathbf{R}_{i j} \cdot \boldsymbol{\mu}_{j}\right)}{R_{i j}^{5}}\right]
\end{aligned}
$$

where $\mathbf{R}_{i j}$ is a vector from atom $i$ to atom $j, q_{i}$ is the charge on atom $i, \boldsymbol{\mu}_{i}$ is the atomic dipole on atom $i$, $\varepsilon_{0}$ is the permittivity of free space, and $\kappa$ is the dielectric constant (taken as 1). The potential energy is summed over all atom pairs in positions 1,4 and higher. Each charge $q_{i}$ is the sum of bond charge increments (BCIs), $q_{i}(b)$,

$$
q_{i}=\sum_{b} q_{i}(b)
$$

where the index $b$ runs over all bonds that contain atom $i$. 
Atomic dipoles are also described in terms of increments associated with bonds. Each of the two atoms of a bond may have a dipole component in the direction of the bond. (The positive direction is determined by the definition of the bond in the force field file. ${ }^{13}$ ) The dipole moment of atom $i$ is then a sum of incremental dipoles, i.e.,

$$
\boldsymbol{\mu}_{i}=\sum_{b} \mu_{i}(b) \mathbf{e}_{b}
$$

where $\mu_{i}(b)$ is the dipole increment of atom $i$ along bond $b$, and $\mathbf{e}_{b}$ is a unit vector along the bond $b$. Again, the index $b$ runs over all bonds that contain atom $i$. This automatically gives the correct symmetry for the dipole of an atom that has several identical bonds. For an end atom, however, which has only one bond, there is the restriction that the dipole moment must be along the bond. Delhommelle et al. ${ }^{14}$ recently used a similar atomic dipole model in alkanes.

Polarization is implemented as a selective combination of induced atomic charges and anisotropically induced atomic dipoles. Thus, under the influence of an electric field, an induced charge

$$
q_{i, \text { ind }}=\sum_{b} 4 \pi \varepsilon_{0} \alpha(b) E_{\mathrm{p}}(b) / r_{b}
$$

is added to the static atomic charge on atom $i$. Here, the index $b$ runs over the bonds containing atom $i$, $\alpha(b)$ is the bond polarizability, $E_{\mathrm{p}}(b)$ is the electric field parallel to the bond (taken as the average of the fields at the atoms of the bond), and $r_{b}$ is the length of bond $b$. The two atoms of a bond get induced charges that are equal in magnitude but opposite in sign. Similarly, an induced atomic dipole

$$
\boldsymbol{\mu}_{i, \text { ind }}=\sum_{b} 4 \pi \varepsilon_{0}\left[\alpha_{\mathrm{p}, i}(b) \mathbf{E}_{\mathrm{p}, i}(b)+\alpha_{\mathrm{pp}, i}(b) \mathbf{E}_{\mathrm{pp}, i}(b)\right]
$$

is added to the static atomic dipole on atom $i . \mathbf{E}_{\mathrm{p}, i}(b)$ and $\mathbf{E}_{\mathrm{pp}, i}(b)$ are, respectively, the parallel and perpendicular (with respect to the bond $b$ ) electric fields at atom $i$, and $\alpha_{\mathrm{p}, i}(b)$ and $\alpha_{\mathrm{pp}, i}(b)$ are the parallel and cylindrically isotropic perpendicular (with respect to the bond $b$ ) atomic polarizabilities, respectively. Thus, each atom of a given bond can have two atomic polarizability parameters associated with it: one describing the polarizability along the bond, and the other describing the polarizability in all directions perpendicular to the bond. This atomic polarizability model has the same symmetry advantages as the fixed atomic dipole model of eq. (3). However, it also imposes cylindrically symmetric polarizability on end atoms. Because the electric field depends on the induced charges and dipoles, and vice versa, an iterative procedure is needed to compute these quantities. In keeping with the usual MM treatment of nonbonded interactions, we only include contributions to the electric field from atoms in positions 1,4 and higher. In this way we also avoid the "polarization catastrophe" resulting from interactions between too closely interacting induced dipoles. ${ }^{15,16}$

In addition to fixed and induced atomic charges and dipoles, the model also encompasses charge and dipole fluxes, i.e., charges and dipoles that depend on the geometry. ${ }^{7,}{ }^{17}$ However, these quantities are not used in this study, because the NMA intramolecular structure is kept fixed. We have not incorporated intermolecular charge transfer in the model.

The parameters are determined by fitting to the $a b$ initio electric potential at grid points around the molecules. We use GAMESS ${ }^{18}$ to generate the points, and to compute the potential, on carefully chosen planes near or through the molecules. The planes are typically chosen to go through all symmetrically nonequivalent bonds or sites in mutually perpendicular directions. Any number of planes necessary for a reliable determination of the parameters can be defined. Although this is less automated than the generic schemes, it is convenient for studying features of special interest in the electric potential, such as lone pair electrons, hydrogen bonds, and polarization. By plotting equipotential lines on these planes, it is easy to compare the $a b$ initio ones with those produced by the optimized parameters. This gives a better picture of the quality of the fit to the electric potential than the rms deviation alone.

The polarizability parameters are also determined by fitting to $a b$ initio electric potentials. In this case external electric fields covering a suitable range of magnitude and direction are applied so as to effectively probe all the different bond and atomic polarizabilities.

The electric potential, $v$, at a point $\mathbf{x}_{j}$, taken to be outside the molecule's van der Waals envelope, is

$$
v\left(\mathbf{x}_{j}\right)=\sum_{i} \frac{1}{4 \pi \varepsilon_{0}}\left[\frac{q_{i}+q_{i, \text { ind }}}{x_{i j}}+\frac{\left(\boldsymbol{\mu}_{i}+\boldsymbol{\mu}_{i, \text { ind }}\right) \cdot \mathbf{x}_{i j}}{x_{i j}^{3}}\right]
$$

where $\mathbf{x}_{i j}$ is a vector from atom $i$ to the point $\mathbf{x}_{j}$. The optimization of atomic charge and dipole parameters is a (one-step) linear least-squares fit if polarization is not included. When polarizability is used, however, the optimization becomes nonlinear (iterative) in the general case because the electric field depends on the optimized (static and induced) 
charges and dipoles, which are unknown prior to the optimization.

Care always should be taken to make sure that the optimized parameters are well defined and not distorted, or rendered indeterminate, by correlations. Therefore, we calculate their statistical uncertainties and correlations using the covariance matrix. ${ }^{19}$ This reveals the cases where the number or kind of data are insufficient for a reliable determination of all the parameters in the optimization.

The merit function used in the optimization to the $\mathrm{ab}$ initio electric potential is the standard $\chi^{2}$ expression,

$$
\chi^{2}=\sum_{i}^{N} w_{i}^{2}\left(v_{i, a b \text { initio }}-v_{i, \text { model }}\right)^{2}
$$

where $N$ is the number of points and $w_{i}$ is a weighting factor. In these calculations the weight of each point was set equal to the distance from the point to the nearest atom. The goodness of fit is given as a weighted relative root-mean-square (wrrms) value,

$$
\text { wrrms }=\sqrt{\frac{\chi^{2}}{\sum_{i} w_{i}^{2} v_{i, a b \text { initio }}^{2}}} .
$$
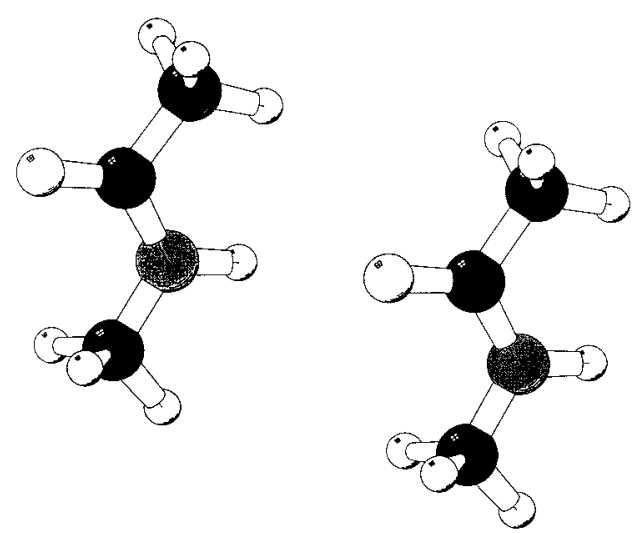

(a)

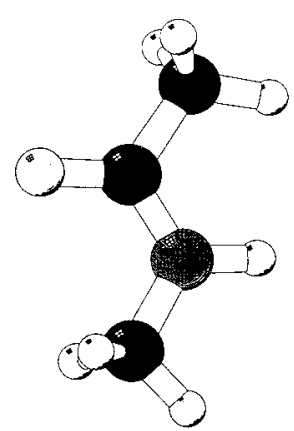

\section{Computational Details}

In two of the dimer configurations considered, the NMA molecules are coplanar (parallel and antiparallel configurations), and in the third the molecules are in mutually orthogonal planes (perpendicular configuration) (Fig. 1). The O $\cdots H$ hydrogen bond distance was set to $2.0 \AA$, which is close to the equilibrium value in these configurations. ${ }^{20}$

The full geometry optimization of NMA was performed using Gaussian $98^{21}$ at the MP2/6$31++G(d, p)$ level of theory, as in our recent calculations on water, formaldehyde, water dimers, and water-formaldehyde complexes. ${ }^{7}$ The same level was used in the GAMESS calculations of the electric potentials. These were calculated with GAMESS because of its built-in option for deriving the potentials on selected planes through the molecule. The geometries of the NMA molecules in the dimers were not optimized but were constrained to be the same as in the isolated molecule. This was done for two reasons. First, the complete SDFF, with a van der Waals potential compatible with our electrostatic parameters, is not yet available. Second,
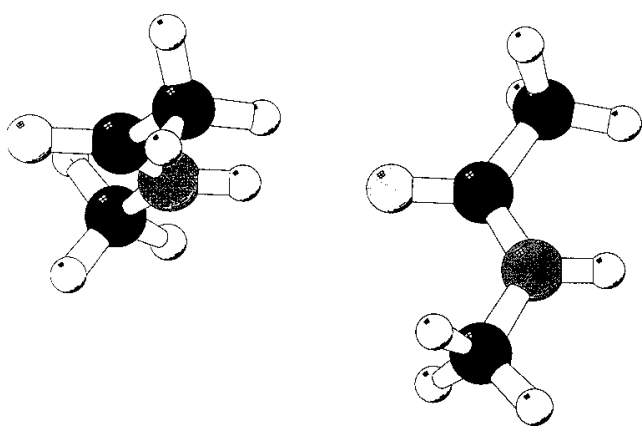

(b)

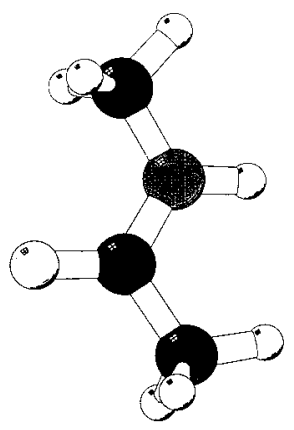

(c)

FIGURE 1. The NMA dimer configurations used in this study. (a) parallel, (b) perpendicular, (c) antiparallel. 
the changes in the electric potential (compared to the electric potential of the monomer) are now due only to induced effects, and are not brought about by changes in the intramolecular structures. All parameter optimizations and other $\mathrm{MM}$ calculations were done with the SPEAR program. ${ }^{13}$

\section{Results and Discussion}

The main objective of this article is to evaluate the ability of our electrostatic model to properly describe electrostatic effects of hydrogen bonding between peptide groups, taking NMA molecules to represent the peptide groups. In the following, we first discuss the determination of parameters for isolated NMA and then their application to the hydrogen-bonded NMA dimers. The parameters to be optimized are a selection of bond charge increments $q_{i}(b)$ of eq. (2), dipole increments $\mu_{i}(b)$ of eq. (3), bond polarizabilities $\alpha(b)$ of eq. (4), and atomic polarizabilities $\alpha_{\mathrm{p}, i}(b)$ and $\alpha_{\mathrm{pp}, i}(b)$ of eq. (5).

A word is in order about the way the results of the parameter optimizations, collected in Tables I-IV, are organized. Table I gives the goodness of fit achieved with each model. Table II shows the bond charge increments (BCIs) obtained with the different methods. The BCIs are interesting to compare separately from the other parameters because they are, in this case, the basic building blocks
TABLE I.

Weighted Relative Root-Mean-Square (wrrms)

Deviations (in \%) of the Reproduction of the MP2/6-31++G(d,p) Electric Potentials of NMA and NMA Dimers.

\begin{tabular}{lcccccc}
\hline & \multicolumn{2}{c}{ Nonpolarizable } & & \multicolumn{2}{c}{ Polarizable ${ }^{\mathrm{a}}$} \\
\cline { 2 - 3 } \cline { 5 - 6 } & Monomer $^{\mathrm{a}}$ & Dimer $^{\mathrm{b}}$ & & POL1 & POL2 \\
\hline NMA & 4.2 & & & 3.6 & 3.7 \\
NMA dimers & 14.5 & $(4.5)^{\mathrm{C}}$ & & 5.2 & 4.1 \\
$\quad$ parallel & 14.7 & $4.1(4.2)^{\mathrm{C}}$ & & 5.1 & 3.9 \\
perpendicular & 14.3 & $4.8(4.8)^{\mathrm{C}}$ & & 5.5 & 4.4 \\
antiparallel & 14.3 & $4.2(4.3)^{\mathrm{C}}$ & & 4.7 & 4.0 \\
\hline
\end{tabular}

a Optimized to NMA monomer.

b Optimized to NMA dimers.

${ }^{c}$ Values in parenthesis are from the simultaneous optimization to all three NMA dimers.

of the MM molecular charge distribution. Table III shows the complete sets of optimized parameters of the two polarizable models, and Table IV gives the actual atomic charges and dipoles that these parameters produce in NMA.

As in our previous study on electrostatic models, ${ }^{7}$ we have calculated electrostatic parameters for two different polarizable models. In the first model (POL1), polarization along the bonds is described by induced atomic charges and polarization perpendicular to the bonds is described by induced

TABLE II.

Optimized Bond Charge Increments, $q_{i}(b)$, (in Electrons) of the Different Bonds ${ }^{a}$ of NMA with Different Electrostatic Models.

$\begin{array}{lllllll}(\mathrm{C}) \mathrm{C}_{\mathrm{m}} \mathrm{H} & (\mathrm{N}) \mathrm{C}_{\mathrm{m}} \mathrm{H} & \mathrm{CC}_{\mathrm{m}} & \mathrm{CN} & \mathrm{NC}_{\mathrm{m}} & \mathrm{CO} & \mathrm{NH}\end{array}$

\begin{tabular}{|c|c|c|c|c|c|c|c|}
\hline \multicolumn{8}{|c|}{ Optimized to NMA } \\
\hline \multicolumn{8}{|l|}{ Nonpolarizable } \\
\hline This work & 0.1886 & 0.0916 & -0.1482 & -0.0649 & 0.1798 & -0.5692 & 0.3265 \\
\hline CHELPG & $0.1278^{b}$ & $0.0606^{b}$ & -0.1121 & -0.0610 & 0.1990 & -0.5860 & 0.3212 \\
\hline \multicolumn{8}{|l|}{ Polarizable } \\
\hline POL1 & 0.1776 & 0.1443 & -0.1925 & -0.0969 & 0.1029 & -0.6766 & 0.3984 \\
\hline POL2 & 0.2410 & 0.1267 & -0.3512 & -0.3744 & 0.2566 & -0.9414 & 0.4581 \\
\hline \multicolumn{8}{|c|}{ Optimized to NMA dimers (nonpolarizable model of this work) ${ }^{\mathrm{C}}$} \\
\hline Parallel & 0.1750 & 0.1284 & -0.1168 & 0.0147 & 0.1174 & -0.6053 & 0.3165 \\
\hline Perpendicular & 0.1738 & 0.1248 & -0.1044 & 0.0415 & 0.1073 & -0.5933 & 0.2990 \\
\hline Antiparallel & 0.1867 & 0.1239 & -0.1387 & -0.0155 & 0.1367 & -0.6219 & 0.3353 \\
\hline All dimers & 0.1459 & 0.1316 & -0.0872 & 0.0159 & 0.1092 & -0.5950 & 0.3160 \\
\hline
\end{tabular}

a Methyl carbon is denoted by $\mathrm{C}_{\mathrm{m}}$.

${ }^{b}$ Mean value of the three $\mathrm{C}_{\mathrm{m}} \mathrm{H}$ BCls of the methyl group.

${ }^{\mathrm{c}}$ The parameters of POL1 and POL2 turned out to be readily transferable from the NMA monomer to the dimers, and were not separately optimized to the dimers. 
TABLE III.

\section{Electrostatic Parameters ${ }^{\mathrm{a}}$ of the Polarizable Models of NMA.}

\begin{tabular}{|c|c|c|}
\hline & POL1 & POL2 \\
\hline $\mathrm{q}\left[(\mathrm{C}) \mathrm{C}_{\mathrm{m}} \mathrm{H}\right]$ & 0.1776 (154) & $0.2410(318)$ \\
\hline $\mathrm{q}\left[(\mathrm{N}) \mathrm{C}_{\mathrm{m}} \mathrm{H}\right]$ & $0.1443(160)$ & 0.1267 (162) \\
\hline $\mathrm{q}\left[\mathrm{CC}_{\mathrm{m}}\right]$ & $-0.1925(148)$ & $-0.3512(229)$ \\
\hline $\mathrm{q}[\mathrm{CN}]$ & $-0.0969(130)$ & $-0.3744(183)$ \\
\hline $\mathrm{q}\left[\mathrm{NC}_{\mathrm{m}}\right]$ & 0.1029 (167) & $0.2566(213)$ \\
\hline $\mathrm{q}[\mathrm{CO}]$ & $-0.6766(92)$ & $-0.9414(270)$ \\
\hline $\mathrm{q}[\mathrm{NH}]$ & $0.3984(114)$ & $0.4581(116)$ \\
\hline$\alpha\left[(\mathrm{C}) \mathrm{C}_{\mathrm{m}} \mathrm{H}\right]$ & $0.6470(1661)$ & \\
\hline$\alpha\left[(\mathrm{N}) \mathrm{C}_{\mathrm{m}} \mathrm{H}\right]$ & $0.6238(705)$ & \\
\hline$\alpha\left[\mathrm{CC}_{\mathrm{m}}\right]$ & $0.7648(4441)$ & \\
\hline$\alpha[\mathrm{CN}]$ & $2.5656(1325)$ & \\
\hline$\alpha\left[\mathrm{NC}_{\mathrm{m}}\right]$ & 2.0941 (2424) & \\
\hline$\alpha[\mathrm{CO}]$ & 3.1064 (2035) & \\
\hline$\alpha[\mathrm{NH}]$ & $1.1364(2587)$ & \\
\hline$\alpha_{\mathrm{p}, \mathrm{H}}\left[(\mathrm{C}) \mathrm{C}_{\mathrm{m}} \mathrm{H}\right]$ & & 0.2900 (912) \\
\hline$\alpha_{\mathrm{p}, \mathrm{C}_{\mathrm{m}}}\left[\mathrm{CC}_{\mathrm{m}}\right]$ & & $0.6193(1911)$ \\
\hline$\alpha_{\mathrm{p}, \mathrm{N}}[\mathrm{CN}]$ & & 1.9449 (1176) \\
\hline$\alpha_{\mathrm{p}, \mathrm{c}}[\mathrm{CO}]$ & & $1.0734(4546)$ \\
\hline$\alpha_{\mathrm{p}, \mathrm{O}}[\mathrm{CO}]$ & & $1.5381(1484)$ \\
\hline$\alpha_{\mathrm{p}, \mathrm{N}}[\mathrm{NH}]$ & & 2.1069 (3419) \\
\hline$\alpha_{\mathrm{pp}, \mathrm{C}_{\mathrm{m}}}\left[(\mathrm{C}) \mathrm{C}_{\mathrm{m}} \mathrm{H}\right]$ & 0.4782 (1269) & 0.5465 (906) \\
\hline$\alpha_{\mathrm{pp}, \mathrm{C}_{\mathrm{m}}}\left[(\mathrm{N}) \mathrm{C}_{\mathrm{m}} \mathrm{H}\right]$ & 0.0 & 0.4073 (333) \\
\hline$\alpha_{\mathrm{pp}, \mathrm{o}}[\mathrm{CO}]$ & 1.0275 (987) & 1.4098 (843) \\
\hline$\alpha_{\mathrm{pp}, \mathrm{N}}[\mathrm{NH}]$ & 1.8969 (1124) & $1.8286(1094)$ \\
\hline
\end{tabular}

a The abbreviations are as follows: $\mathrm{q}$ refers to static $\mathrm{BCl}, \alpha$ to bond polarizability, and $\alpha_{\mathrm{p}}$ and $\alpha_{\mathrm{pp}}$ to parallel and perpendicular atomic polarizability, respectively. The subscripts $O$, $\mathrm{C}, \mathrm{C}_{\mathrm{m}}, \mathrm{N}$, and $\mathrm{H}$ refer to oxygen, $\mathrm{sp}^{2}$ carbon, $\mathrm{sp}^{3}$ carbon, nitrogen, and hydrogen atoms, and the letters in brackets to the corresponding bonds. The following sign convention is used: $q(A B)$ is added to atom $B$ and $-q(A B)$ is added to atom $A$. Numbers in parenthesis are statistical uncertainties of the last digits.

atomic dipoles. In the second model (POL2), both the parallel and perpendicular polarizations are represented by induced atomic dipoles. The parameters of the polarizable models were optimized for the isolated NMA molecule in the presence of various (up to $\pm 1 \mathrm{~V} / \AA$ ) external electric fields. Parameters of nonpolarizable models have also been optimized separately for the isolated NMA molecule and for the NMA dimers. In the optimizations to the dimers the same kind and number of parameters were used as in the NMA monomer (i.e., the parameters are common for both monomers in the dimers).

\section{ISOLATED NMA}

Nonpolarizable models consisting of fixed BCIs and atomic dipoles were first studied. It turned out that a pure charge model reproduced the electric potential of NMA very satisfactorily (wrrms $=4.2 \%$, see Table I). By adding a few fixed atomic dipoles, slightly better fits were achieved, with wrrms deviations of $2.4-2.8 \%$. However, in this case some of the parameters tended to be strongly correlated and sometimes indeterminate, despite the large number of data points used (seven planes and about 6000 grid points on each plane). Thus, such atomic dipoles are not unique and are not very reliable. We therefore decided to accept the point charge model for the description of the electrostatic interactions in NMA. The charge parameters (BCIs) of the nonpolarizable model are given in Table II. It is interesting to note that the N-methyl and the C-methyl C-H BCIs differ by about a factor of 2 . The electric potentials given by these parameters and by $a b$ initio are shown in Figure 2. As can be seen from the figure, the strongly polar peptide group dominates the electric potential. The lone pair electrons on the oxygen and nitrogen atoms do not appear to affect the electric equipotential lines in any significant way at distances associated with nonbonded interactions ( $\sim 1.8 \AA$ or longer). Close to the nuclei (1.0-1.2 $\AA$ ), however, the electric potentials show some possible signs of the lone pairs. On the other hand, the $\pi$-electron system of the peptide $\mathrm{CN}$ bond seems not to contribute to the electric potential, even at close distances. The reason may be that this contribution is hidden by the large negative charge of the nitrogen atom.

The charge parameters of the peptide group obtained with our model are close to those given by the (MP2) CHELPG scheme ${ }^{21}$ (see Table II), whereas the Mulliken population analysis ${ }^{21}$ gives much less polar bonds for the NMA peptide group. Our model yields more positively charged methyl hydrogen atoms, and therefore, more negatively charged methyl carbon atoms than the CHELPG method (see Table IV). However, the CHELPG BCIs can well be used as initial values for the charge parameters in NMA. (As previously mentioned, when polarizability is included, the optimization is nonlinear and, therefore, requires initial parameter values.) The wrrms deviation of the CHELPG electric potential from the $a b$ initio values on the planes used to optimize our charge parameters is $5.7 \%$, which is comparable to that of our charge model.

The charge and polarizability parameters of the polarizable models were then optimized for NMA 
Atomic Charges (e) and Dipoles (eÅ) in NMA with the Different Methods Studied.

\begin{tabular}{|c|c|c|c|c|c|c|}
\hline \multirow[b]{3}{*}{ Atom $^{a}$} & \multicolumn{2}{|c|}{ Nonpolarizable } & \multicolumn{4}{|c|}{ Polarizable } \\
\hline & \multirow{2}{*}{$\frac{\text { This Work }}{\text { Charge }}$} & \multirow{2}{*}{$\frac{\text { CHELPG }}{\text { Charge }}$} & \multicolumn{2}{|c|}{ POL1 } & \multicolumn{2}{|c|}{ POL2 } \\
\hline & & & Charge & Dipole & Charge & Dipole \\
\hline (C) $C_{m}$ & -0.714 & -0.495 & -0.655 & 0.056 & -1.074 & 0.134 \\
\hline $\mathrm{H}^{\mathrm{b}}$ & 0.189 & 0.138 & 0.169 & 0 & 0.241 & 0.037 \\
\hline $\mathrm{H}$ & 0.189 & 0.108 & 0.144 & 0 & 0.241 & 0.045 \\
\hline $\mathrm{H}^{\mathrm{b}}$ & 0.189 & 0.138 & 0.169 & 0 & 0.241 & 0.037 \\
\hline C & 0.782 & 0.759 & 0.867 & 0 & 1.667 & 0.005 \\
\hline $\mathrm{O}$ & -0.569 & -0.586 & -0.598 & 0.019 & -0.941 & 0.193 \\
\hline $\mathrm{N}$ & -0.572 & -0.581 & -0.561 & 0.099 & -1.089 & 0.270 \\
\hline $\mathrm{H}$ & 0.327 & 0.321 & 0.372 & 0 & 0.458 & 0 \\
\hline$(\mathrm{N}) \mathrm{C}_{\mathrm{m}}$ & -0.095 & 0.017 & -0.371 & 0 & -0.124 & 0.152 \\
\hline $\mathrm{H}$ & 0.092 & 0.059 & 0.132 & 0 & 0.127 & 0 \\
\hline $\mathrm{H}^{\mathrm{b}}$ & 0.092 & 0.061 & 0.165 & 0 & 0.127 & 0 \\
\hline $\mathrm{H}^{\mathrm{b}}$ & 0.092 & 0.061 & 0.165 & 0 & 0.127 & 0 \\
\hline
\end{tabular}

a Methyl carbon is denoted by $\mathrm{C}_{\mathrm{m}}$.

b Out-of-plane atom.

by using electric potentials calculated with and without external electric fields. The number of planes with different electric potentials thereby increased to 28 , each of them containing about 6000 grid points. The external electric fields were chosen
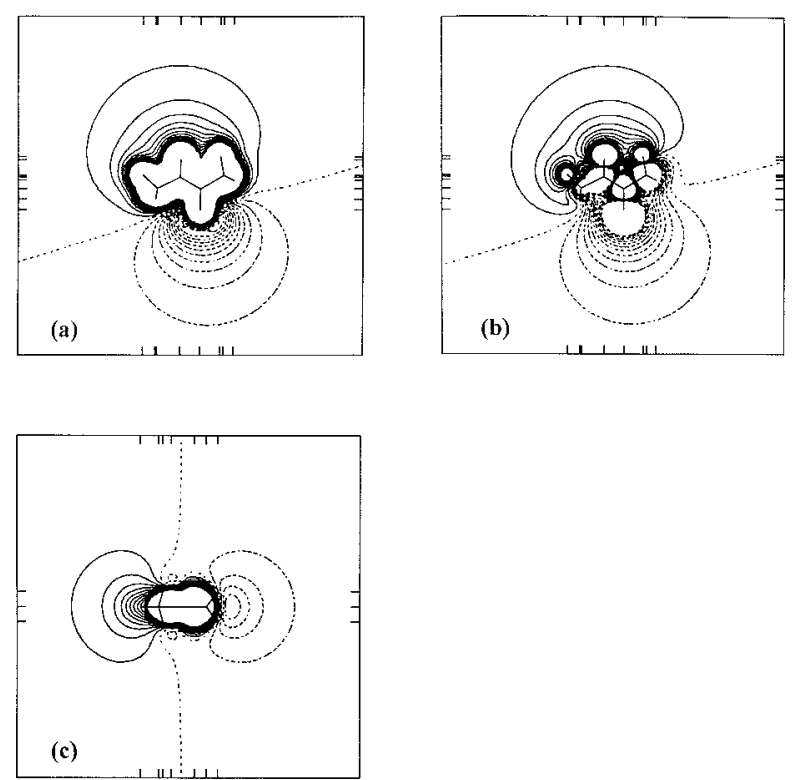

FIGURE 2. $A b$ initio MP2/6-31++G(d,p) and MM electric equipotential lines of NMA (a) ab initio in-plane, (b) atomic charge model in-plane, (c) ab initio out-of-plane (through peptide CN bond). Solid line: positive potential; broken line: negative potential; dotted line: zero potential. in several directions: along a bond of the peptide group, perpendicular to the bond but in the molecular plane, and perpendicular to the molecular plane. These electric fields effectively probe the polarizability of all bonds and atoms in NMA. Due to the internal electric field in NMA, the charge parameters (BCIs) are different in the polarizable and nonpolarizable models (see Table II). All parameters (including the ones shown in Table II) of the polarizable models are given in Table III, and the wrrms deviations of the electric potentials from the ab initio values are given in Table I. Both POL1 and POL2 perform excellently, with wrrms deviations of 3.6 and $3.7 \%$, respectively. Both models use perpendicular atomic polarizability on the carbon atom of the C-methyl $\mathrm{CH}$ bonds (in POL2 also on the carbon atom of the $\mathrm{N}$-methyl $\mathrm{CH}$ bonds), on the oxygen atom of the $\mathrm{C}=\mathrm{O}$ bond, and on the nitrogen atom of the $\mathrm{NH}$ bond. These parameters are similar in POL1 and POL2 because they mainly determine the polarizability perpendicular to the molecular plane. The static BCIs are completely different in POL1 and POL2, especially for the $\mathrm{CC}, \mathrm{C}=\mathrm{O}, \mathrm{CN}$ (peptide), and NC bonds. This is due to the induced in-plane atomic dipoles on the heavy atoms in POL2, which are absent in POL1. In practice, POL1 was easier to parameterize than POL2, because POL1 has only one parameter for parallel polarizability of each bond. The optimization of POL1 therefore does not as readily become unstable and result in indeterminate parameters as does the optimization of POL2. POL1 is also more unique, because the polarizabil- 
TABLE V.

Calculated Molecular Dipole Moments (in Debye) of NMA and NMA Dimers with Different Electrostatic Models and by ab initio.

\begin{tabular}{|c|c|c|c|c|c|}
\hline & \multicolumn{2}{|c|}{ Nonpolarizable } & \multicolumn{2}{|c|}{ Polarizable ${ }^{\mathrm{a}}$} & \multirow[b]{2}{*}{$\mathrm{MP} 2 / 6-31++\mathrm{G}(\mathrm{d}, \mathrm{p})$} \\
\hline & Monomer $^{a}$ & Dimer $^{b}$ & POL1 & POL2 & \\
\hline NMA & 4.00 & & 3.98 & 4.00 & 4.00 \\
\hline \multicolumn{6}{|l|}{ NMA dimers } \\
\hline Parallel & 8.01 & 9.34 & 9.70 & 9.66 & 9.39 \\
\hline Perpendicular & 7.82 & 9.06 & 9.51 & 9.47 & 9.17 \\
\hline Antiparallel & 7.64 & 8.91 & 9.30 & 9.24 & 8.99 \\
\hline
\end{tabular}

a Using parameters optimized to the NMA monomer.

b Using parameters optimized to the NMA dimers.

ity of each bond can always be determined. In POL2, on the other hand, the bond polarizabilities have to be replaced by a non-unique subset of all possible atomic polarizabilities along the bonds. Of course, as regards the atomic polarizabilities perpendicular to the bonds, both POL1 and POL2 are nonunique. However, POL1 is easier to use also in this case because there are smaller correlations between the bond polarizabilities and the perpendicular atomic polarizabilities than there are between the parallel and perpendicular atomic polarizabilities.

It is interesting to note that the polarizable models also reproduce the electric potential of the isolated NMA molecule slightly better than does the nonpolarizable fixed charge model (see Table I). This is due to the fact that the polarizable models allow for induced atomic dipoles, and in the case of POL1 for slightly different charges on the methyl hydrogen atoms. Table IV shows the actual atomic charges and dipoles in NMA as given by the different methods. As can be seen, the POL1 charges on the C-methyl in-plane and out-of-plane hydrogens differ by $\sim 0.025 \mathrm{e}$, and on the N-methyl hydrogens by $\sim 0.033 \mathrm{e}$. The CHELPG charges show a similar difference for the C-methyl but not for the N-methyl group. Many of the induced atomic dipoles are also sizable, especially the POL2 ones. Not surprisingly, such explicit intramolecular polarization helps to better account for the electric potential of NMA. This also is the reason why the charge parameters in the fixed charge model are different from those of POL1 and POL2, especially the BCIs of the N-methyl $\mathrm{CH}$ and of the $\mathrm{C}=\mathrm{O}$ bonds (see Table II). The results of the polarizable models are not quite as good as the best ones given by the nonpolarizable charge/dipole model, which implicitly includes the induced intramolecular effects but which, because of severe correlations, could not be optimized uniquely for NMA.

Regarding the other electrostatic molecular properties studied here, namely molecular dipole moments and molecular polarizability, they also are well reproduced by our polarizable models. POL1 calculates the molecular dipole moment to be 3.98 debye (D), and POL2 gives $4.00 \mathrm{D}$ (see Table V). The $a b$ initio value is $4.00 \mathrm{D}$. The experimental value is $3.73 \mathrm{D}^{22}$ The CHELPG charges give $4.10 \mathrm{D}$ for the dipole moment.

The molecular polarizability of NMA is rather anisotropic. The diagonal elements of the polarizability tensor are given in Table VI. The values of these elements are very close to each other in POL1 and POL2, and are also in good agreement with the $a b$ initio ones.

\section{NMA DIMERS}

The $a b$ initio electric potentials of the hydrogenbonded NMA dimers were calculated in 23 planes of the parallel configuration, in 21 planes of the perpendicular configuration, and in 7 planes of the antiparallel configuration. The number of grid points in each plane varied from $\sim 5200$ to $\sim 6200$. Nonpolarizable electrostatic models with fixed parameters are used in most force fields for proteins. We, therefore, first transferred the static model with fixed charge parameters, optimized to the isolated NMA molecule, to the different dimer configurations. As can be seen in Figure 3, the results are in severe disagreement with the $a b$ initio electric potentials of the dimers, yielding wrrms deviations of $14-15 \%$ (see Table I). This indicates that the changes in the electric potentials caused by induced effects in the different configurations of the peptide groups are large enough to essentially make the fixed charge model 
TABLE VI.

Diagonal Elements of Calculated Polarizability Tensors $\left(\AA^{3}\right)$ of NMA and NMA Dimers.

\begin{tabular}{|c|c|c|c|c|c|c|c|c|c|c|c|c|}
\hline & \multicolumn{3}{|c|}{ NMA } & \multicolumn{3}{|c|}{ Parallel Dimer } & \multicolumn{3}{|c|}{ Perpendicular Dimer } & \multicolumn{3}{|c|}{ Antiparallel Dimer } \\
\hline & $\mathrm{MP}^{\mathrm{a}}$ & POL1 & POL2 & $\mathrm{MP} 2^{\mathrm{b}}$ & POL1 & POL2 & $M P 2^{b}$ & POL1 & POL2 & $\mathrm{MP} 2^{\mathrm{b}}$ & POL1 & POL2 \\
\hline$x x$ & 8.08 & 8.30 & 8.03 & 15.19 & 15.58 & 14.98 & 12.87 & 13.04 & 12.65 & 15.77 & 16.60 & 16.45 \\
\hline yy & 7.49 & 7.46 & 7.45 & 10.23 & 9.95 & 9.71 & 13.01 & 12.97 & 12.56 & 14.14 & 14.34 & 13.89 \\
\hline $\mathrm{zz}$ & 5.41 & 5.19 & 5.09 & 16.64 & 17.54 & 17.47 & 16.16 & 17.03 & 16.91 & 12.08 & 12.02 & 11.70 \\
\hline
\end{tabular}

$\mathrm{a}_{6}-31++\mathrm{G}(\mathrm{d}, \mathrm{p})$.

b $6-31+G(d)$.

nontransferable from NMA. To find out whether a set of fixed charges can be tailored for the hydrogenbonded dimers, we specifically optimized BCIs for the different configurations of the dimer (see Table II and Fig. 3c). In these individually optimized nonpolarizable models the induced effects are implicitly accounted for, and the wrrms deviations are indeed small $(\sim 4-5 \%)$. This is, however, achieved at the cost of transferability of the parameters, and except for the $\mathrm{N}$-methyl $\mathrm{CH}$ and the $\mathrm{C}=\mathrm{O} \mathrm{BCI}$, the parameters change from one configuration to another, some of the changes being as large as $\sim 30 \%$ (see Table II). By reoptimizing the (monomer-defined) BCIs for all dimer configurations simultaneously the wrrms deviation became $4.5 \%$ and the charge
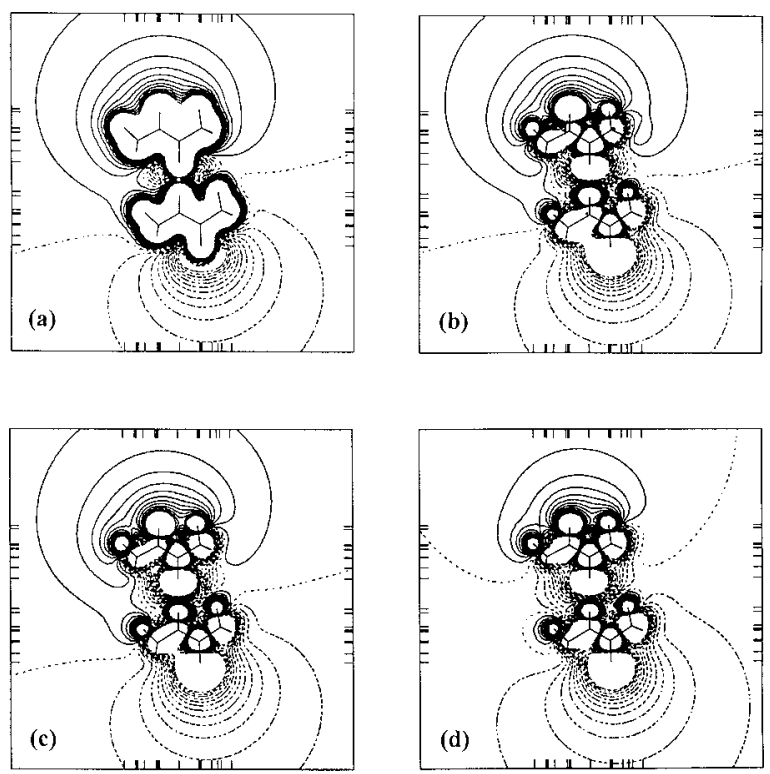

FIGURE 3. $A b$ initio MP2/6-31++G(d,p) and MM electric equipotential lines of the parallel configuration of the NMA dimer: (a) ab initio, (b) POL1, (c) reoptimized nonpolarizable dimer-specific charge model; (d) charge model transferred from NMA monomer. Line designations as in Figure 2. parameters then correspond to average values for all the hydrogen-bonded configurations studied. This is an excellent fit, but the BCIs are significantly different from those of the monomer, indicating nontransferability when the intermolecular distance varies. There are also other reasons for caution. We found in our previous study ${ }^{7}$ that there are some hydrogen-bonded configurations in which the electric potential cannot be properly accounted for by nonpolarizable models even if the parameters are specifically optimized to those configurations. On the other hand, the monomer-optimized polarizable models proved to work excellently in all configurations studied. A polarizable model can also continuously account for the electric potential when the intermolecular distances and configurations vary, as in molecular dynamics and Monte Carlo simulations.

The polarizable models, with induced charges and induced atomic dipoles (POL1) or with induced atomic dipoles only (POL2), optimized to the isolated NMA molecule (parameters shown in Table III) were directly transferred to all the dimer configurations. As seen in Figure 3 and Table I, the agreement with the $a b$ initio electric potentials is excellent. The results given by the two polarizable models differ only slightly from each other. POL1 reproduces the electric potential of the parallel NMA dimer with a wrrms deviation of $5.1 \%$, whereas POL2 gives 3.9\%. The corresponding values for the perpendicular configuration are 5.5 and $4.4 \%$, and for the antiparallel configuration 4.7 and $4.0 \%$. POL1 reproduces the ab initio electric potentials of all three NMA dimers with a wrrms deviation of $5.2 \%$ and POL2 gives $4.1 \%$.

The molecular dipole moment of each dimer came out about the same with POL1 and POL2 and, as seen in Table $\mathrm{V}$, are slightly overestimated but are within $3-4 \%$ of the $a b$ initio values. The nonpolarizable charge model of NMA, though, underestimates the dimer dipole moments by $\sim 15 \%$. The individu- 
ally optimized dimer-specific (nonpolarized) BCIs, on the other hand, yield dipole moments within $1 \%$ of the $a b$ initio values. Using the charge parameters obtained from the simultaneous optimization of the parallel, perpendicular and antiparallel configurations yields about the same results, or 9.30, 9.10, and $8.91 \mathrm{D}$ for the dipole moments of these dimers, respectively. The corresponding $a b$ initio values are $9.39,9.17$, and $8.99 \mathrm{D}$. All of the studied methods predict the same trend, viz., that the parallel configuration has the largest molecular dipole moment and the antiparallel configuration has the smallest one.

As for the isolated NMA molecule, the molecular polarizabilities of the NMA dimers also are rather anisotropic. The diagonal elements of the polarizability tensors, presented in Table VI, are similar in POL1 and POL2 and are within $\sim 5 \%$ of the $a b$ initio values. Because of disk space limitations we could not calculate the polarizabilities for the dimers using the MP2/6-31++G(d,p) level. The values given for the dimers in Table VI are instead MP2/6-31+G(d) values. They are, however, not expected to be very different from those of MP2/6-31++G(d,p), because for isolated NMA the $\mathrm{MP} 2 / 6-31+\mathrm{G}(\mathrm{d})$ polarizabilities are only about $1 \%$ lower than the MP2 $/ 6-31++G(d, p)$ ones.

Regarding the intermolecular electrostatic potential energies of the dimers, the results are analogous to those of our previous study. ${ }^{7}$ As can be seen from Table VII, the polarizable models give potential energies that are much more attractive than those obtained by the nonpolarizable models. POL1 yields slightly more attractive energies than POL2 (by $0.5-0.6 \mathrm{kcal} / \mathrm{mol}$ ). The potential energies given by the nonpolarizable charge model transferred from the NMA monomer are the least attractive, being $\sim 5 \mathrm{kcal} / \mathrm{mol}$ higher (i.e., less negative) than those of the polarizable models. Even the

\section{TABLE VII.}

\section{Calculated Intermolecular Electrostatic Energies $(\mathrm{kcal} / \mathrm{mol})$ of NMA Dimers with the Different Electrostatic Models.}

\begin{tabular}{lcclll}
\hline \multirow{2}{*}{ NMA dimer } & \multicolumn{2}{c}{ Nonpolarizable } & & \multicolumn{2}{c}{ Polarizable $^{\mathrm{a}}$} \\
\cline { 2 - 3 } \cline { 5 - 6 } & Monomer $^{\mathrm{a}}$ & Dimer $^{\mathrm{b}}$ & & POL1 & POL2 \\
\hline Parallel & -6.44 & -7.97 & & -11.71 & -11.08 \\
Perpendicular & -6.53 & -8.37 & -11.79 & -11.27 \\
Antiparallel & -6.37 & -7.99 & & -11.68 & -11.21 \\
\hline
\end{tabular}

\footnotetext{
a Using parameters optimized to the NMA monomer.

b Using parameters optimized to the NMA dimers.
}

reoptimized dimer-specific nonpolarizable (NMA) BCIs give $3-4 \mathrm{kcal} / \mathrm{mol}$ higher energies than the polarizable models. Because the potential energy due to polarization is always attractive, it is not surprising that the polarizable models yield lower energies. However, the magnitudes of the energy differences are perhaps larger than expected, especially when considering that the dimer-specific charges (which implicitly account for the polarization) reproduce the $a b$ initio electric potentials and dipole moments very satisfactorily. Therefore, in a nonpolarizable energy function, the polarization energy has to be compensated by artificially more attractive (or less repulsive) van der Waals interactions.

\section{Conclusions}

We have shown that polarization contributes very significantly to the electric potentials of a set of hydrogen-bonded NMA dimers. This indicates that polarization is also important in hydrogen bonding between peptide groups in proteins, and needs to be taken explicitly into account in maximally transferable models. Although a nonpolarizable atomic charge model turned out to be nontransferable from the NMA monomer to the dimers, the monomerbased polarizable models studied here were found to reproduce well the $a b$ initio electric potential, dipole moment, and polarizability of each dimer. This indicates that there may not be a need to include intermolecular charge transfer explicitly in the model (although this has been suggested recently ${ }^{23}$ ). Without the use of polarizability, however, good agreement with the $a b$ initio electric potential and dipole moments of the dimers could only be obtained if the charge parameters were directly optimized to the dimer configurations. Even then, the intermolecular electrostatic potential energies were $3-4 \mathrm{kcal} / \mathrm{mol}$ higher than those given by the polarizable models.

Two polarizable models were tested. The first one (POL1) uses induced bond charge increments (bond polarizability) to account for polarization along the bonds, and cylindrically isotropic induced atomic dipoles to account for polarization perpendicular to the bonds. The second model (POL2) uses (different) induced atomic dipoles to describe polarizability both parallel and perpendicular to the bonds. POL1 and POL2 reproduced the ab initio data about equally well but it turned out that, in practice, POL1 was easier to optimize than POL2. This is because the bond polarizability parameters of POL1 can all be determined more or less uniquely, whereas in POL2 a nonunique subset of atomic polarizability parameters has to be selected to avoid 
severe correlations between the parameters. POL1 also has another advantage in that it allows charge flow along the molecular chains. Because of their accurate descriptions of the electrostatic properties also in hydrogen-bonded systems, both POL1 and POL2 constitute a physically reasonable basis for the development of spectroscopically determined polarizable force fields.

\section{Acknowledgments}

The research was also supported in part by NSF Cooperative Agreement ACI-9619020 through computing resources provided by the National Partnership for Advanced Computational Infrastructure at the San Diego Supercomputer Center.

\section{References}

1. Berendsen, H. J. C. Science 1998, 282, 642.

2. Palmo, K.; Pietila, L.-O.; Krimm, S. J Comput Chem 1991, 12, 385.

3. Palmo, K.; Pietila, L.-O.; Krimm, S. Comput Chem 1993, 17, 67.

4. Palmo, K.; Mirkin, N. G.; Pietila, L.-O.; Krimm, S. Macromolecules 1993, 26, 6831.

5. Palmo, K.; Mirkin, N. G.; Krimm, S. J Phys Chem A 1998, $102,6448$.

6. Mannfors, B.; Sundius, T.; Palmo, K.; Pietila, L.-O.; Krimm, S. J Mol Struct 2000, 521, 49.

7. Mannfors, B.; Palmo, K.; Krimm, S. J Mol Struct 2000, 556, 1.

8. Applequist, J.; Carl, J. R.; Fung, K.-K. J Am Chem Soc 1972, 94, 2952.

9. Rick, S. W.; Stuart, S. J.; Berne, B. J. J Chem Phys 1994, 101, 6141.

10. Stern, H. A.; Kaminski, G. A.; Banks, J. L.; Zhou, R.; Berne, B. J.; Friesner, R. A. J Phys Chem B 1999, 103, 4730.
11. Ma, B.; Lii, J.-H.; Allinger, N. L. J Comput Chem 2000, 21, 813.

12. Winn, P. J.; Ferenzy, G. G.; Reynolds, C. A. J Comput Chem 1999, 20, 704.

13. Palmo, K.; Krimm, S. SPEAR, a program for molecular mechanics and dynamics, to appear.

14. Delhommelle, J.; Granucci, G.; Brenner, V.; Millié, P.; Boutin, A.; Fuchs, A. H. Mol Phys 1999, 97, 1117.

15. Thole, B. T. Chem Phys 1981, 59, 341.

16. Caldwell, J. W.; Kollman, P. A. J Phys Chem 1995, 99, 6208.

17. Palmo, K.; Krimm, S. J Comput Chem 1998, 19, 754.

18. Schmidt, M. W.; Baldridge, K. K.; Boatz, J. A.; Elbert, S. T.; Gordon, M. S.; Jensen, J. H.; Koseki, S.; Matsunaga, N.; Nguyen, K. A.; Su, S. J.; Windus, T. L.; Dupuis, M.; Montgomery, J. A. J Comput Chem 1993, 14, 1347.

19. Press, W. H.; Flannery, B. P.; Teukolsky, S. A.; Vetterling, W. T. Numerical Recipes; Cambridge University Press: Cambridge, MA, 1987.

20. Qian, W.; Mirkin, N. G.; Krimm, S. Chem Phys Lett 1999, $315,125$.

21. Frisch, M. J.; Trucks, G. W.; Schlegel, H. B.; Scuseria, G. E.; Robb, M. A.; Cheeseman, J. R.; Zakrzewski, V. G.; Montgomery, J. A., Jr.; Stratmann, R. E.; Burant, J. C.; Dapprich, S.; Millam, J. M.; Daniels, A. D.; Kudin, K. N.; Strain, M. C.; Farkas, O.; Tomasi, J.; Barone, V.; Cossi, M.; Cammi, R.; Mennucci, B.; Pomelli, C.; Adamo, C.; Clifford, S.; Ochterski, J.; Petersson, G. A.; Ayala, P. Y.; Cui, Q.; Morokuma, K.; Malick, D. K.; Rabuck, A. D.; Raghavachari, K.; Foresman, J. B.; Cioslowski, J.; Ortiz, J. V.; Stefanov, B. B.; Liu, G.; Liashenko, A.; Piskorz, P.; Komaromi, I.; Gomperts, R.; Martin, R. L.; Fox, D. J.; Keith, T.; Al-Laham, M. A.; Peng, C. Y.; Nanayakkara, A.; Gonzalez, C.; Challacombe, M.; Gill, P. M. W.; Johnson, B.; Chen, W.; Wong, M. W.; Andres, J. L.; Gonzalez, C.; Head-Gordon, M.; Replogle, E. S.; Pople, J. A. Gaussian 98, Revision A.3; Gaussian, Inc.: Pittsburgh, PA, 1998.

22. Lide, D. R., Ed. CRC Handbook of Chemistry and Physics; CRC Press: Boca Raton, FL, 1992-1993; 73rd ed.

23. van der Vaart, A.; Bursulaya, B. D.; Brooks, C. L., III; Merz, K. M., Jr. J Phys Chem B 2000, 104, 9554. 